\title{
Treatment of severe acute pancreatitis via endoscopic pancreatic stenting and nasopancreatic drainage: Case reports
}

\author{
ZUOZHENG WANG ${ }^{*}$, QI WANG ${ }^{*}$, JIANJUN SONG, WEIJIE YAO, PENG LEI, \\ CHAOFENG TANG, PENG YUAN and JUNZHI LENG

\begin{abstract}
Department of Hepatobiliary Surgery, The General Hospital of Ningxia Medical University, Yinchuan, Ningxia 750004, P.R. China
\end{abstract}

Received June 20, 2018; Accepted November 6, 2018

DOI: $10.3892 /$ etm.2018.6958

\begin{abstract}
Severe acute pancreatitis (SAP) is associated with high mortality. SAP is generally treated by conservative management at the early phase, and removal of the pancreatic and peripancreatic necrotic tissue at the late phase. However, studies have suggested that the surgical treatment of SAP should focus on pressure reduction and drainage. In this case report, 3 SAP patients of 44, 30 and 60 years of age were treated at the General Hospital of Ningxia Medical University. They underwent emergency endoscopic pancreatic stenting at the early phase and nasopancreatic drainage at the late phase when peripancreatic encapsulated effusion was observed. All patients were successfully treated and discharged from the hospital. The disease duration of the patients was 71,58 , and 88 days, respectively. Our cases suggested that the surgical strategy of endoscopic pancreatic stenting at the early phase and nasopancreatic drainage at the late phase is promising for the treatment of SAP.
\end{abstract}

\section{Introduction}

Severe acute pancreatitis (SAP) is an acute, severe pancreatic inflammation with a mortality rate of $30 \%$ (1). Currently, the management of SAP remains controversial, primarily due to poor understanding of its pathogenesis. Generally, SAP patients undergo conservative management at the early phase, and removal of the peripancreatic necrotic tissue at the late phase (2). Nevertheless, recent studies have suggested that the incidence of SAP is associated with pancreatic duct obstruction

Correspondence to: Dr Zuozheng Wang, Department of Hepatobiliary Surgery, The General Hospital of Ningxia Medical University, 804 South Victory Street, Yinchuan, Ningxia 750004, P.R. China

E-mail: zt767d@163.com

${ }^{*}$ Contributed equally

Key words: severe acute pancreatitis, ERCP, pancreatic stenting, nasopancreatic drainage, mortality and hypertension, and hence surgical treatment of SAP should focus on pressure reduction and drainage (3). Herein, we present the cases of 3 SAP patients who were successfully treated in 2016 via endoscopic pancreatic stenting at the early phase and nasopancreatic drainage at the late phase.

\section{Case reports}

Ethics approval. The present study was approved by the Research Ethics Committee of the General Hospital of Ningxia Medical University (Yinchuan, China). Each patient signed an informed consent for permission to use the clinical data and images.

Case 1. A 44-year-old male was admitted to the General Hospital of Ningxia Medical University due to severe upper abdominal pain, heavy sweat and increased respiratory and heart rate. The patient had elevated biliary and liver enzyme level, white blood cell (WBC) count of $20.90 \times 10^{9} / 1$, neutral granulocyte ratio (NEUT\%) of $81.1 \%$, amylase of 3,879.6 U/1, lipase of 20,000 U/1, APACHE II score of 10, and oxygenation index of 149. The patient was diagnosed with severe acute biliary pancreatitis. He underwent emergency endoscopic operation. Endoscopic sphincterotomy (EST) was performed. A wire was placed into the pancreatic duct and a 6-cm-long 6 -Fr-sized stent was inserted over the wire. A large amount of pancreatic fluid containing protein plugs was removed. A second wire was placed into the bile duct, and a nasobiliary duct was placed along the guide wire. The patient was then given non-invasive ventilation and conventional infusion therapy for anti-infection, acid suppression, inhibition of enzyme secretion and liver protection. The patient had restored respiratory function and urination, WBC of $11.88 \times 10^{9} / 1$ and NEUT\% of $90.6 \%$ on day 1 postoperatively, and began oral feeding on day 7. Computed tomography (CT) scan showed progressive pancreatic necrosis and acute peripancreatic fluid accumulation (Fig. 1a and b). However, the patient developed fever and increased WBC, and his condition was not improved by anti-infection treatment. The pancreatic duct stent was suspected to be blocked, and endoscopic operation was performed again on day 14 to replace the old pancreatic duct stent with an 8-cm-long 7-Fr-sized stent. WBC dropped to $12.07 \times 10^{\%} / 1$, and NEUT\% was $88.8 \%$ 

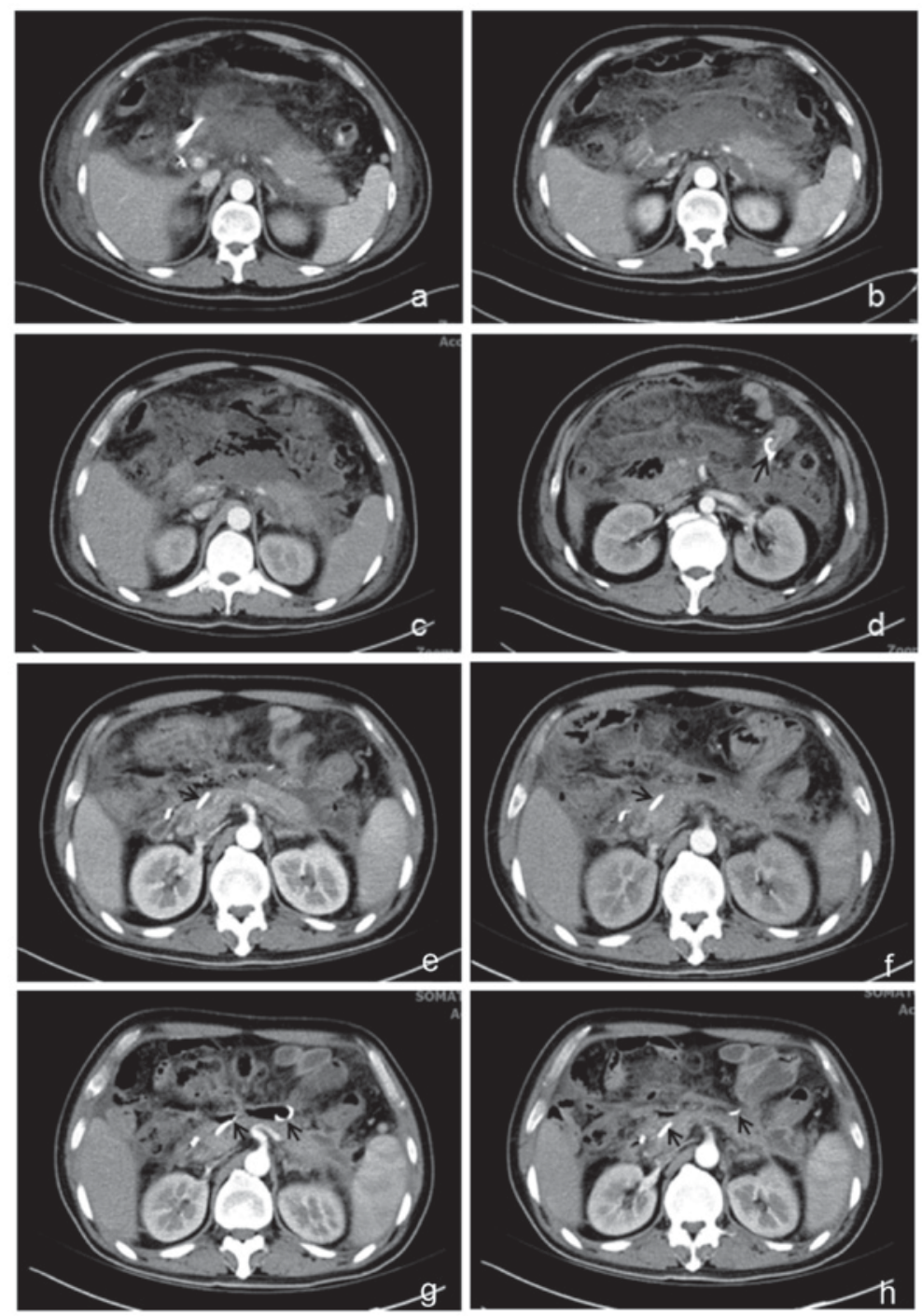

Figure 1. Abdominal enhanced CT of a 44-year-old male SAP patient (case 1) (a) at 5 days after the first pancreatic duct stenting showing extensive pancreatic necrosis; (b) at 2 weeks after the first pancreatic duct stenting showing aggravated pancreatic necrosis and peripancreatic fluid; (c and d) at 1 week after the second pancreatic duct stenting showing peripancreatic infectious necrosis and the pancreatic duct stent (marked by the black arrow) that has slipped into the duodenum; (e) at 12 days, (f) 17 days, (g) 30 days and (h) 45 days after the second nasopancreatic drainage showing progressively decreased peripancreatic infectious necrosis. The nasopancreatic duct is marked by a black arrow. CT, computed tomography; SAP, severe acute pancreatitis.
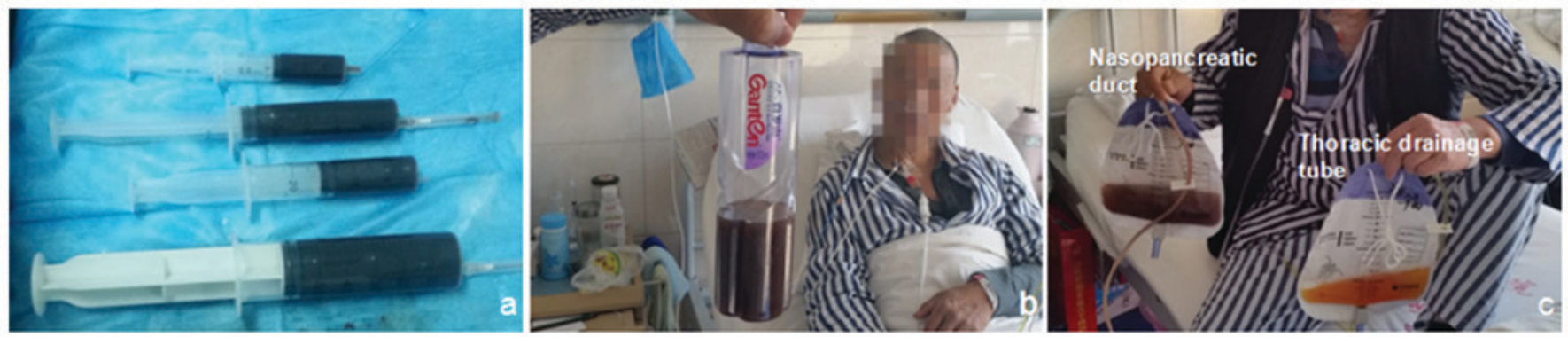

Figure 2. Images showing the amount and color of pancreatic fluid from a 44-year-old male SAP patient (case 1) (a) during the second nasopancreatic drainage, (b) at 1 day, and (c) at 1 week after the surgery. SAP, severe acute pancreatitis.

immediately after the operation. At 1 week after the second stenting, abdominal enhanced CT showed peripancreatic encapsulated effusion and infective necrosis. The pancreatic duct stent had slipped into duodenum (Fig. 1c and d). The patient then underwent nasopancreatic drainage. Purulent pancreatic fluid $(30 \mathrm{ml})$ was removed during the operation and daily postoperatively $(10-30 \mathrm{ml})$. The patient was given antibiotics treatment according to the antibiotic susceptibility of bile cultures. The nasopancreatic duct fell off on day 12 after the first nasopancreatic duct drainage, and 
the patient underwent endoscopic surgery to insert a new nasopancreatic duct. Dark red purulent pancreatic fluid was removed during the surgery $(100 \mathrm{ml}$, Fig. $2 \mathrm{a})$ and daily postoperatively (50-200 ml, Fig. 2b). The fluid became clear after 1 week (Fig. 2c). CT scan showed the gradually decreased peripancreatic infection necrosis (Fig. 1e-h). Patient was cured and discharged at 28 days after the last surgery. The disease duration was 71 days.

Case 2. A 30-year-old female was admitted to the hospital due to 19-day obvious abdominal pain. The patient had fever $\left(38.5^{\circ} \mathrm{C}\right)$, oliguria, increased heart rate and breathing, WBC of $11.67 \times 10^{9} / 1$, NEUT\% of $77.9 \%$, oxygenation index of 181, and APACHE II score of 8 . CT scan revealed acute necrotizing pancreatitis with extensive peripancreatic fluid. The patient was diagnosed with severe acute idiopathic pancreatitis, and underwent endoscopic operation to place a 6-cm-long 5-Fr-sized pancreatic duct stent and a nasobiliary duct. Pancreatic fluid containing protein plugs was removed. The patient was given conventional infusion treatment. On day 1 postoperatively, patient's blood test, amylase and lipase levels, respiratory and heart rate, and oxygen saturation returned to normal range. APACHE II score was 2. On day 2 , the patient was allowed oral feeding. On day 3 , the patient developed intermittent fever and increased blood parameters, which were gradually returned to normal range after a 5-day anti-infection therapy. CT suggested acute necrotizing pancreatitis with markedly alleviated pancreatic fluid collection. The patient was discharged from the hospital on day 8 as per her own request, but was admitted back into our hospital due to severe abdominal pain and high fever $\left(39^{\circ} \mathrm{C}\right)$ on day 13 . The patient had WBC of $34.23 \times 10^{9} / 1$, NEUT\% of $89.4 \%$, and APACHE II score of 13. CT scan suggested necrotizing pancreatitis with peripancreatic encapsulated effusion and infective necrosis. She underwent endoscopic surgery to remove the pancreatic duct stent and to perform nasopancreatic drainage. Chylous pancreatic fluid was removed during the operation $(120 \mathrm{ml})$ and daily postoperatively (50-100 ml/day). She was given postoperative anti-infection therapy and non-invasive ventilation (oxygenation index of 198). On day 1 after drainage, the patient had greatly relieved abdominal pain, WBC of $14.15 \times 10^{9} / 1$, NEUT\% of $78.9 \%$, APACHE II score of 7, and normal respiratory and heart rate. The patient was allowed oral feeding on day 2 . The respiratory function was gradually restored, and the body temperature and blood test results gradually went back to normal range. CT scan suggested progressive reduction of peripancreatic infection necrosis and complete removal of pancreatic fluid collections on day 30 after drainage. The tube was pulled out and the patient was discharged from the hospital. The disease duration was 58 days, including 34 days of hospitalization.

Case 3. A 60-year-old female with serum amylase of 1,493.6 U/1, WBC of $10.06 \times 10^{9} / 1$, NEUT $\%$ of $90.5 \%$, APACHE II score of 8 , and oxygenation index of 179 was diagnosed with severe acute idiopathic pancreatitis in the hospital. She underwent emergency endoscopic operation immediately after admission to place a pancreatic duct stent and a nasobiliary duct. Pancreatic fluid containing protein plugs were removed.
The patient was given postoperative conventional infusion therapy and non-invasive ventilation. Respiratory function and urination were gradually restored. Blood results and body temperature returned to normal, and the patient began oral feeding on day 12 . On day 22, the patient developed high fever and elevated WBC despite the anti-infection therapy. CT scan suggested peripancreatic encapsulated effusion. The patient then underwent nasopancreatic drainage. A large amount of white pus $(100 \mathrm{ml})$ was removed during the operation and daily postoperatively (50-150 ml). Abdominal enhanced CT scan at day 7,14 and 30 showed that peripancreatic encapsulated effusion was clearly reduced. The patient was discharged after 68 days of hospitalization. The disease duration was 88 days since the onset.

\section{Discussion}

Traditionally, the best surgical intervention timing for SAP is at 4 weeks after onset when walled-off necrosis (WON) and infection has developed (4). The surgical intervention includes percutaneous drainage or endoscopic drainage followed by infectious necrotic tissue removal. We treated 3 SAP patients via endoscopic pancreatic stenting at the early phase and nasopancreatic drainage at the late phase, and achieved great outcomes. Our cases suggested that the indicated surgical regime might be a promising strategy for the treatment of SAP.

Organ failure induced by severe systemic inflammatory response is known as the most important cause of death in the early phase of SAP (4). Therefore, the main treatment goal at this stage is to control the systemic inflammatory response. Patients are generally treated by conservative infusion because early surgical intervention often aggravate the inflammatory response, leading to an increased mortality rate $(5,6)$. Endoscopic operation does not create extra trauma or cause the diffusion of peripancreatic fluid, and thus is operable even in SAP patients with severe organ dysfunction (7). More importantly, we found viscous pancreatic fluid or even 'protein plugs' which, if not removed, might clog the duct and aggravate the conditions. In this study, after pancreatic duct obstruction was removed by surgery, the inflammatory indexes (APACHE II score, WBC, body temperature, heart rate) were improved in all patients, suggesting that the clearance of pancreatic duct obstruction is beneficial for the early control of systemic inflammatory response in SAP.

Most aseptic necrotizing pancreatitis can be cured by conservative treatments. Nevertheless, peripancreatic infectious necrosis occurs in $25-70 \%$ of SAP patients (6), and has become the primary cause of death in the late phase of SAP (8). Currently, a step-up approach of percutaneous drainage is the main surgical approach (4). However, approaches such as transmural or percutaneous image guided drainage (9), and necrotic tissue removal via abdominal or video assisted retroperitoneal debridement (VARD) (10) need to create an additional channel for the drainage of infection necrosis. Reportedly, 31-44\% of patients with acute necrotizing pancreatitis had pancreatic duct rupture (11-13). For these patients, drainage through the duodenal papillary pancreatic duct can enter the necrotic effusion cavity directly through the ruptured pancreatic duct, so as to achieve the drainage without an additional wound. Studies 
have shown that this method can improve the cure rate of peripancreatic hydrops, prevent recurrence of peripancreatic hydrops and repair the pancreatic duct rupture (14-16), and reduce the risk of hemorrhage and digestive tract leakage caused by a puncture (17).

In the current 3 cases, nasopancreatic duct drainage was performed and a large amount of infection necrosis was removed during and after the surgery. Patients' conditions were greatly improved, and inflammation and peripancreatic infectious necrosis were gradually alleviated and eventually disappeared, indicating that this surgical intervention is feasible for the treatment of late necrotizing pancreatitis. Imaging findings suggested that the infection necrotic cavities were linked to the nasopancreatic duct, which might explain the efficacy of nasopancreatic duct drainage in infection necrotizing pancreatitis.

Although the pathogenesis of acute pancreatitis has not been fully elucidated yet, studies have suggested pancreatic duct obstruction and hypertension as key events of both biliary and non-biliary acute pancreatitis $(18,19)$. Moreover, pancreatic duct hypertension is positively correlated with the severity of acute pancreatitis (20), leading to pancreatic ischemia necrosis and severe pancreatitis $(21,22)$. Consistently, our cases had pancreatic duct obstruction during both early and late phase, confirming the critical role of pancreatic duct obstruction and hypertension in SAP. A prompt and effective drainage of the pancreatic duct reduced the intra-duct pressure, and thereby greatly improved the healing of SAP. None of the 3 patients were aggravated by pancreatitis after ERCP. We did not perform pancreatogram during the operation, because acute pancreatitis was associated with damage to the main pancreatic duct and gland vesicle, and pressure on the pancreatic duct during pancreatogram could directly lead to rupture of the main pancreatic duct or gland vesicle, thus inducing postoperative pancreatitis after ERCP and aggravating acute pancreatitis $(23,24)$. The clear diagnosis of the 3 patients led to avoidance of unnecessary pancreatic duct angiography and reduced the incidence of pancreatitis after ERCP. Pancreatic duct stenting is an important prevention method for postoperative pancreatitis after ERCP, and even an important remedy for postoperative pancreatitis after ERCP (25). In our study, pancreatic duct catheterization was used to treat acute pancreatitis, and these two factors prevented the occurrence of postoperative pancreatitis.

In summary, we reported on 3 SAP cases that were successfully treated via pancreatic stent at the early phase and nasopancreatic drainage at the late phase. Results suggested that the indicated treatment strategy might alleviate the systemic inflammatory reaction via removal of pancreatic duct obstruction, and thus improve the healing of SAP, although multicenter clinical trials are needed.

\section{Acknowledgements}

Not applicable.

\section{Funding}

This study was supported by the Technology Support Program of Ningxia (2015KJHM40).

\section{Availability of data and materials}

The datasets used and/or analyzed during the current study are available from the corresponding author on reasonable request.

\section{Authors' contributions}

ZW and QW interpreted the CT results. JS and WY acquired and analyzed the general data of the patients. PL, ZW and CT performed the surgery. PY and JL were responsible for the inflammatory indexes analysis. All authors read and approved the final manuscript.

\section{Ethics approval and consent to participate}

The present study was approved by the Research Ethics Committee of the General Hospital of Ningxia Medical University (Yinchuan, China). Each patient signed an informed consent for permission to use the clinical data and images.

\section{Patient consent for publication}

All patients signed an informed consent for permission to use the clinical data and images.

\section{Competing interests}

The authors declare that they have no competing interests.

\section{References}

1. Beger HG and Rau BM: Severe acute pancreatitis: Clinical course and management. World J Gastroenterol 13: 5043-5051, 2007.

2. Mier J, León EL, Castillo A, Robledo F and Blanco R: Early versus late necrosectomy in severe necrotizing pancreatitis. Am J Surg 173: 71-75, 1997.

3. Tang C, Wang B, Xie B, Liu H and Chen P: Treatment of severe acute pancreatitis through retroperitoneal laparoscopic drainage. Front Med 5: 302-305, 2011.

4. van Santvoort HC, Besselink MG, Bakker OJ, Hofker HS, Boermeester MA, Dejong CH, van Goor H, Schaapherder AF, van Eijck CH, Bollen TL, et al; Dutch Pancreatitis Study Group: A step-up approach or open necrosectomy for necrotizing pancreatitis. N Engl J Med 362: 1491-1502, 2010.

5. Zerem E, Imamović G, Sušić A and Haračić B: Step-up approach to infected necrotising pancreatitis: A 20 -year experience of percutaneous drainage in a single centre. Dig Liver Dis 43: 478-483, 2011.

6. van Baal MC, van Santvoort HC, Bollen TL, Bakker OJ, Besselink MG and Gooszen HG; Dutch Pancreatitis Study Group: Systematic review of percutaneous catheter drainage as primary treatment for necrotizing pancreatitis. Br J Surg 98: 18-27, 2011.

7. Uhl W, Warshaw A, Imrie C, Bassi C, McKay CJ, Lankisch PG Carter R, Di Magno E, Banks PA, Whitcomb DC, et al; International Association of Pancreatology: IAP guidelines for the surgical management of acute pancreatitis. Pancreatology 2: 565-573, 2002 .

8. Besselink MG, van Santvoort HC, Boermeester MA, Nieuwenhuijs VB, van Goor H, Dejong CH, Schaapherder AF and Gooszen HG; Dutch Acute Pancreatitis Study Group: Timing and impact of infections in acute pancreatitis. Br J Surg 96: 267-273, 2009.

9. Hollemans RA, van Brunschot S, Bakker OJ, Bollen TL, Timmer R, Besselink MG and van Santvoort HC; Dutch Pancreatitis Study Group: Minimally invasive intervention for infected necrosis in acute pancreatitis. Expert Rev Med Devices 11: 637-648, 2014. 
10. Horvath K, Freeny P, Escallon J, Heagerty P, Comstock B, Glickerman DJ, Bulger E, Sinanan M, Langdale L, Kolokythas O, et al: Safety and efficacy of video-assisted retroperitoneal debridement for infected pancreatic collections: A multicenter, prospective, single-arm phase 2 study. Arch Surg 145: 817-825, 2010.

11. Neoptolemos JP, London NJ and Carr-Locke DL: Assessment of main pancreatic duct integrity by endoscopic retrograde pancreatography in patients with acute pancreatitis. Br J Surg 80: 94-99, 1993.

12. Lau ST, Simchuk EJ, Kozarek RA and Traverso LW: A pancreatic ductal leak should be sought to direct treatment in patients with acute pancreatitis. Am J Surg 181: 411-415, 2001.

13. Uomo G, Molino D, Visconti M, Ragozzino A, Manes G and Rabitti PG: The incidence of main pancreatic duct disruption in severe biliary pancreatitis. Am J Surg 176: 49-52, 1998.

14. Trevino JM, Tamhane A and Varadarajulu S: Successful stenting in ductal disruption favorably impacts treatment outcomes in patients undergoing transmural drainage of peripancreatic fluid collections. J Gastroenterol Hepatol 25: 526-531, 2010.

15. Bhasin DK and Rana SS: Combining transpapillary pancreatic duct stenting with endoscopic transmural drainage for pancreatic fluid collections: Two heads are better than one! J Gastroentero Hepatol 25: 433-434, 2010.

16. Shrode CW, Macdonough P, Gaidhane M, Northup PG, Sauer B $\mathrm{Ku}$ J, Ellen K, Shami VM and Kahaleh M: Multimodality endoscopic treatment of pancreatic duct disruption with stenting and pseudocyst drainage: How efficacious is it? Dig Liver Dis 45: 129-133, 2013.

17. Hookey LC, Debroux S, Delhaye M, Arvanitakis M, Le Moine O and Devière J: Endoscopic drainage of pancreatic-fluid collections in 116 patients: A comparison of etiologies, drainage techniques, and outcomes. Gastrointest Endosc 63: 635-643, 2006.
18. Harvey MH, Wedgwood KR, Austin JA and Reber HA: Pancreatic duct pressure, duct permeability and acute pancreatitis. Br J Surg 76: 859-862, 1989

19. Siqin D, Wang C, Zhou Z and Li Y: The key event of acute pancreatitis: Pancreatic duct obstruction and bile reflux, not a single one can be omitted. Med Hypotheses 72: 589-591, 2009.

20. Fujiwara H: Pressure measurement in pancreatic duct and biliary duct system in dogs with acute pancreatitis. Kobe J Med Sci 37: 47-55, 1991.

21. Shi CX, Chen JW, Carati CJ, Schloithe AC, Toouli J and Saccone GT: Effects of acute pancreatic duct obstruction on pancreatic perfusion: Implication of acute pancreatic duct decompression. Scand J Gastroenterol 37: 1328-1333, 2002.

22. Arendt T: Bile-induced acute pancreatitis in cats. Roles of bile, bacteria, and pancreatic duct pressure. Dig Dis Sci 38: 39-44, 1993.

23. Varadarajulu S, Rana SS and Bhasin DK: Endoscopic therapy for pancreatic duct leaks and disruptions. Gastrointest Endosc Clin N Am 23: 863-892, 2013.

24. He QB, Xu T, Wang J, Li YH, Wang L and Zou XP: Risk factors for post-ERCP pancreatitis and hyperamylasemia: A retrospective single-center study. J Dig Dis 16: 471-478, 2015.

25. Shi QQ, Ning XY, Zhan LL, Tang GD and Lv XP: Placement of prophylactic pancreatic stents to prevent post-endoscopic retrograde cholangiopancreatography pancreatitis in high-risk patients: A meta-analysis. World J Gastroenterol 20: 7040-7048, 2014.

This work is licensed under a Creative Commons

Attribution-NonCommercial-NoDerivatives 4.0 International (CC BY-NC-ND 4.0) License. 